\title{
Review
}

\section{Acupuncture Affects Regional Blood Flow in Various Organs}

\section{Sae Uchida and Harumi Hotta}

\author{
Department of the Autonomic Nervous System, Tokyo Metropolitan Institute of Gerontology, Tokyo 173-0015, \\ Japan
}

\begin{abstract}
In this review, our recent studies using anesthetized animals concerning the neural mechanisms of vasodilative effect of acupuncture-like stimulation in various organs are briefly summarized. Responses of cortical cerebral blood flow and uterine blood flow are characterized as nonsegmental and segmental reflexes. Among acupuncture-like stimuli delivered to five different segmental areas of the body; afferent inputs to the brain stem (face) and to the spinal cord at the cervical (forepaw), thoracic (chest or abdomen), lumbar (hindpaw) and sacral (perineum) levels, cortical cerebral blood flow was increased by stimuli to face, forepaw and hindpaw. The afferent pathway of the responses is composed of somatic groups III and IV afferent nerves and whose efferent nerve pathway includes intrinsic cholinergic vasodilators originating in the basal forebrain. Uterine blood flow was increased by cutaneous stimulation of the hindpaw and perineal area, with perineal predominance. The afferent pathway of the response is composed of somatic group II, III and IV afferent nerves and the efferent nerve pathway includes the pelvic parasympathetic cholinergic vasodilator nerves. Furthermore, we briefly summarize vasodilative regulation of skeletal muscle blood flow via a calcitonin gene-related peptide (CGRP) induced by antidromic activation of group IV somatic afferent nerves. These findings in healthy but anesthetized animals may be applicable to understanding the neural mechanisms improving blood flow in various organs following clinical acupuncture.
\end{abstract}

Keywords: axon reflex-cerebral blood flow-muscle blood flow-somato-autonomic reflexuterine blood flow

\section{Introduction}

Somatic afferent stimulation to the skin, muscle and joints has been found to produce reflex responses in visceral function via autonomic efferent nerves in animals anesthetized to eliminate emotional factors (1). Clinical application of somatic afferent nerve stimulation by acupuncture has been successfully employed for the adjustment of autonomic malfunctions (2-4). The neural mechanism of the autonomic responses following acupuncture has now accumulated (5-7). Recent acupuncture studies demonstrated that acupuncture-like stimulation delivered to anesthetized animals produced

For reprints and all correspondence: Sae Uchida, Department of the Autonomic Nervous System, Tokyo Metropolitan Institute of

Gerontology, 35-2 Sakaecho, Itabashi-ku Tokyo 173-0015, Japan.

Tel: 81-3-3964-3241; Fax: 81-3-3579-4776; E-mail: suchida@tmig.or.jp reflex responses of various visceral functions, e.g. gastric motility $(5,8)$, bladder contraction $(5,9)$, cardiovascular responses (10), adrenal medullary hormonal function $(11,12)$, etc. These acupuncture-like stimulation-induced responses have been proven to be reflexes in which afferents are cutaneous and muscle somatic afferent nerves and efferents are autonomic efferent nerves. Some of these somato-autonomic reflexes are segmental spinal reflexes and others are non-segmental supraspinal reflexes $(1,5,6)$.

The possibility that acupuncture improves blood flow of various organs has been suggested as a mechanism of acupuncture effects on visceral functions. For example, it is likely that improvement of cerebral circulation is a possible mechanism of the effect of acupuncture in the treatment of cerebral stroke (13) and vascular dementia $(14,15)$, caused by disturbances in cerebral blood 
flow. Concerning the mechanism of acupuncture effects on female infertility, blood flow of the uterus was expected to increase by acupuncture stimulation (16,17). Concerning the mechanism of acupuncture therapy for neck stiffness, blood flow of the neck muscles was expected to increase by acupuncture stimulation $(4,18)$.

Recently, on the basis of somato-autonomic reflexes, the neural mechanisms of acupuncture effects on regional blood flow in various organs have been examined using anesthetized animals. These studies are introduced in this review article, especially on blood flow of the cerebral cortex, uterus and skeletal muscles.

\section{Non-segmental and Segmental Mechanism of Blood Flow Regulation}

\section{Cortical Cerebral Blood Flow (CBF)}

\section{Acupuncture Increases CBF}

In anesthetized rats, Uchida et al. (19) examined the effects on cortical cerebral blood flow of acupuncture-like stimulation of various segmental areas and further examined the neural mechanisms of the acupunctureinduced responses of $\mathrm{CBF}$. An acupuncture needle (diameter $340 \mu \mathrm{m}$ ) was inserted into the skin and underlying muscles to a depth of $5 \mathrm{~mm}$ and was twisted manually right and left approximately once every second for $1 \mathrm{~min}$. CBF of the parietal cortex was measured using a laser Doppler flowmeter. Acupuncture-like manual stimulation of the face, forepaw and hindpaw produced significant increases in $\mathrm{CBF}$, but stimulation of the chest and perineum did not produce significant responses (Fig. 1). Both ipsilateral and contralateral $\mathrm{CBF}$ responses were almost identical. The increases in $\mathrm{CBF}$ were always accompanied by pressor response of mean arterial pressure (MAP). However, after spinal transection at the first and second thoracic level, the blood pressure response to stimulation of the face and forepaw was suppressed, whereas the increase in CBF still took place. This indicates that the $\mathrm{CBF}$ response is independent of changes in MAP.

\section{Somatic Nerves are the Afferent Pathway of the CBF Response}

The increase in $\mathrm{CBF}$ induced by forepaw stimulation was abolished by severance of the somatic nerves at the brachial plexus. Forepaw stimulation enhanced the activity of the radial, ulnar and median nerves. Furthermore, passing an electric current through acupuncture needles showed that excitation of group III (A $\delta$ ) and group IV (C) afferent fibers in the somatic nerve was capable of producing an increase in $\mathrm{CBF}$, whereas excitation of group I $(A \alpha)$ and group II $(A \beta)$ fibers was ineffective. These findings of $\mathrm{CBF}$ responses during acupuncture-like stimulation is partially a confirmation of the finding of $\mathrm{CBF}$ response to noxious mechanical cutaneous stimulation (pinching) in anesthetized rats reported by Adachi et al. (20).

A

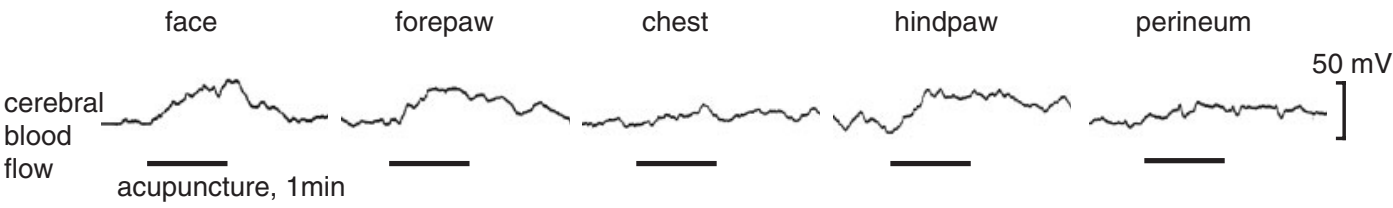

B

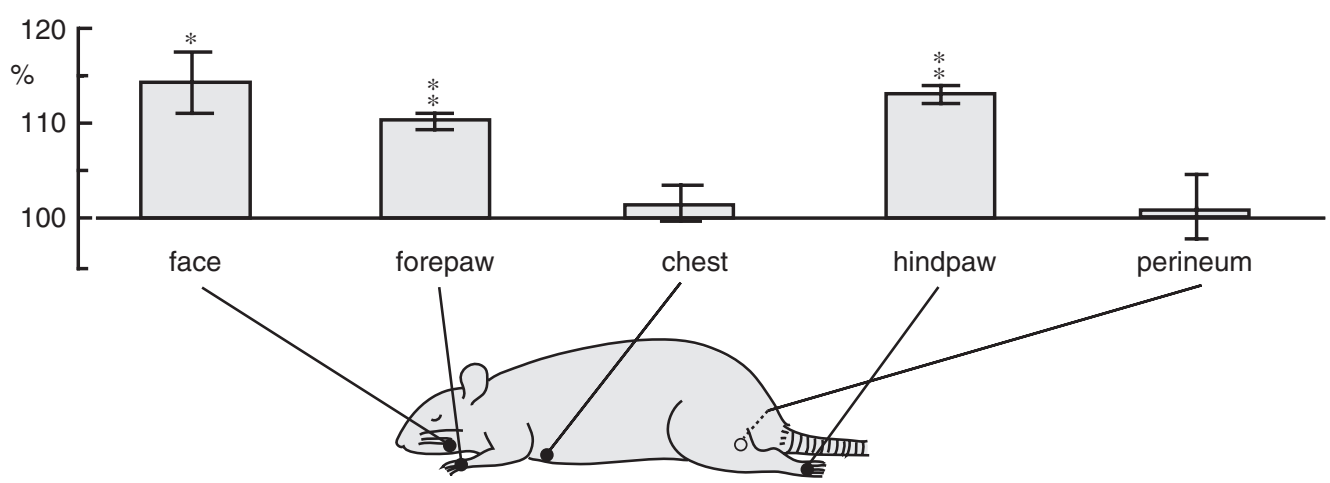

Figure 1. The effect of acupuncture-like stimulation of various areas on CBF in the unilateral parietal cortex in central nervous system-intact rats. (A) Sample recordings of CBF in the parietal cortex ipsilateral to the stimulating site following acupuncture-like stimulation of the face, forepaw, chest, hindpaw and perineum for $1 \mathrm{~min}$ (indicated by horizontal bars). (B) Summary of CBF responses. Each point and vertical bar represent the mean \pm SEM. ${ }^{*} P<0.05$, ${ }^{*} P<0.01$; significantly different from prestimulus control values using a paired $t$-test. Modified from Uchida et al. (19). 
Intracranial Cholinergic Vasodilators are the Efferent Pathway of the CBF Response

Uchida et al. (19) examined involvement of extrinsic autonomic nerves innervating the cerebral blood vessels on the CBF response. The CBF response elicited by electro-acupuncture stimulation of a forepaw in the spinalized rats did not significantly change either (i) after eliminating the sympathetic and parasympathetic nerves innervating the cerebral blood vessels by severance of the cervical sympathetic trunks and removal of the palatine ganglia, bilaterally, or (ii) after i.v. injection of propranolol, a $\beta$-adrenergic receptor blockade, or additional injection of phentolamine, an $\alpha$-adrenergic receptor blockade and (iii) after i.v. injection of methylatropine, or additional injection of hexamethonium, blood brain barrier (BBB), impermeable muscarinic and nicotinic acetylcholine (ACh) receptor antagonists. These evidences neglect an involvement of sympathetic and parasympathetic efferents for the CBF response.

Then, involvement of intrinsic cholinergic fibers originating in the magnocellular nucleus of the basal forebrain [the nucleus basalis of Meynert (NBM)] (21) was also examined. The $\mathrm{CBF}$ responses were attenuated after i.v. injection of atropine, a BBB permeable muscarinic $\mathrm{ACh}$ receptor antagonist and were further attenuated by an additional i.v. injection of mecamylamine, a BBB permeable nicotinic ACh receptor blockade (Fig. 2C-E). Furthermore, extracellular $\mathrm{ACh}$ release in the parietal cortex measured every 3 min using microdialysis (Fig. 2A) and a high-performance liquid chromatography (HPLC) measuring system was increased by acupuncture-like manual stimulation of a forepaw (Fig. 2B). ACh, detected to increase in the cortex, appeared to be released from cholinergic nerve terminals in the cortex following acupuncture-like stimulation. In fact, Akaishi et al. (22) demonstrated the excitation of NBM neurons by cutaneous pinching in anesthetized rats. The $\mathrm{CBF}$ response elicited by electro-acupuncture stimulation of a forepaw in spinalized rats was totally abolished after the lesion of bilateral NBM. Therefore, activation of the intracranial cholinergic system originating in NBM is important for elicitation of an increase in CBF following acupuncture-like stimulation, independent of blood pressure.

\section{Summary of the Reflex Pathway for the CBF Response}

We conclude that the increase in $\mathrm{CBF}$, elicited by acupuncture-like stimulation, is a non-segmental supraspinal reflex response, whose afferent pathway is composed of somatic group III and IV afferent nerves and whose efferent nerve pathway includes intrinsic cholinergic vasodilators originating in the NBM (Fig. 5). The increase in $\mathrm{CBF}$ was produced by stimulation of the face, forepaw or hindpaw, but not by that of chest or perineal area. The difference in response magnitude appears to
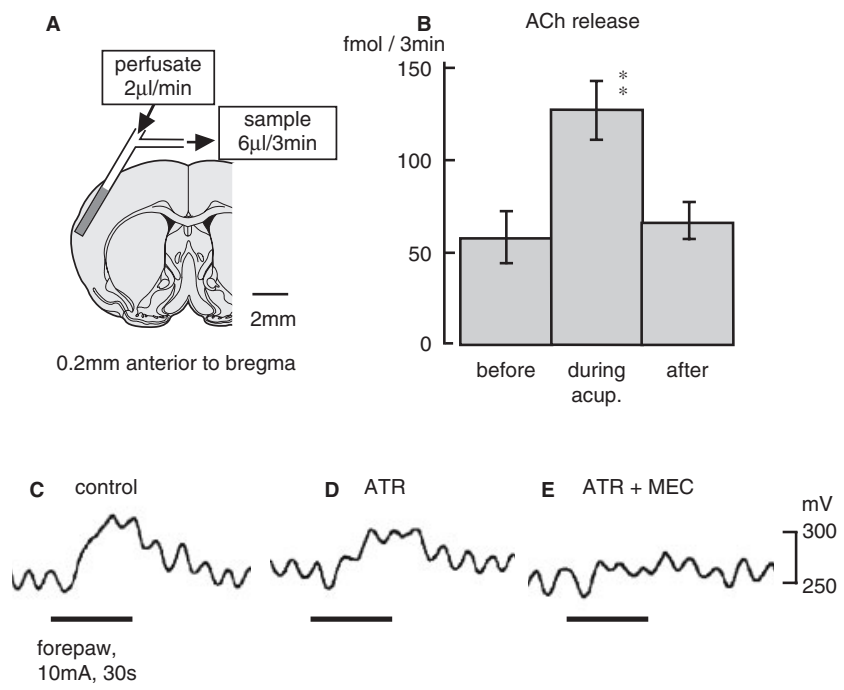

Figure 2. (A) Diagram of the experiment demonstrating the microdialysis probe in the cortex. (B) Effects of acupuncture-like stimulation of the unilateral forepaw on extracellular ACh release in the parietal cortex ipsilateral to the stimulating site in central nervous system-intact rats. Each column and vertical bar show the mean \pm SEM. ${ }^{* *} P<0.01$; significantly different from the prestimulus control value using one-way repeated ANOVA followed by Dunnett's multiple comparison test. (C-E) Effect of cholinergic receptor antagonist permeable to BBB on $\mathrm{CBF}$ response induced by electro-acupuncture stimulation of a forepaw in spinalized rats. ATR: atropine, $5 \mathrm{mg} / \mathrm{kg}$, i.v. MEC: mecamylamine, $20 \mathrm{mg} / \mathrm{kg}$, i.v. Modified from Uchida et al. (19).

depend either on different density of afferent innervations of various segmental skin and muscle or on different connections to the central cholinergic vasodilators, from various segmental areas of the body.

\section{Uterine Blood Flow (UBF)}

\section{Cutaneous Mechanical Stimuli Increase UBF}

It has been reported that electro-acupuncture reduced blood flow impedance in the uterine arteries of infertile women (16). However, neural mechanisms of UBF responses to somatic afferent stimulation have not yet been clarified. Hotta et al. (23) examined the effects of cutaneous mechanical afferent stimulation of various skin areas on UBF in anesthetized non-pregnant rats. Blood flow of the uterine body was measured continuously using a laser Doppler flowmeter. Noxious pinching stimulation of the face, forepaw or abdomen was ineffective, while pinching stimulation of the hindpaw and perineum produced increases in UBF (Fig. 3A). The response after perineal pinching was larger than the response after hindpaw pinching (Fig. 3B). In the case of innocuous brushing stimuli, only the perineal area caused an increase in UBF (Fig. 3C and D). The mean arterial pressure increased following pinching of the face, forepaw, hindpaw and perineum by $5-30 \mathrm{mmHg}$, but the 


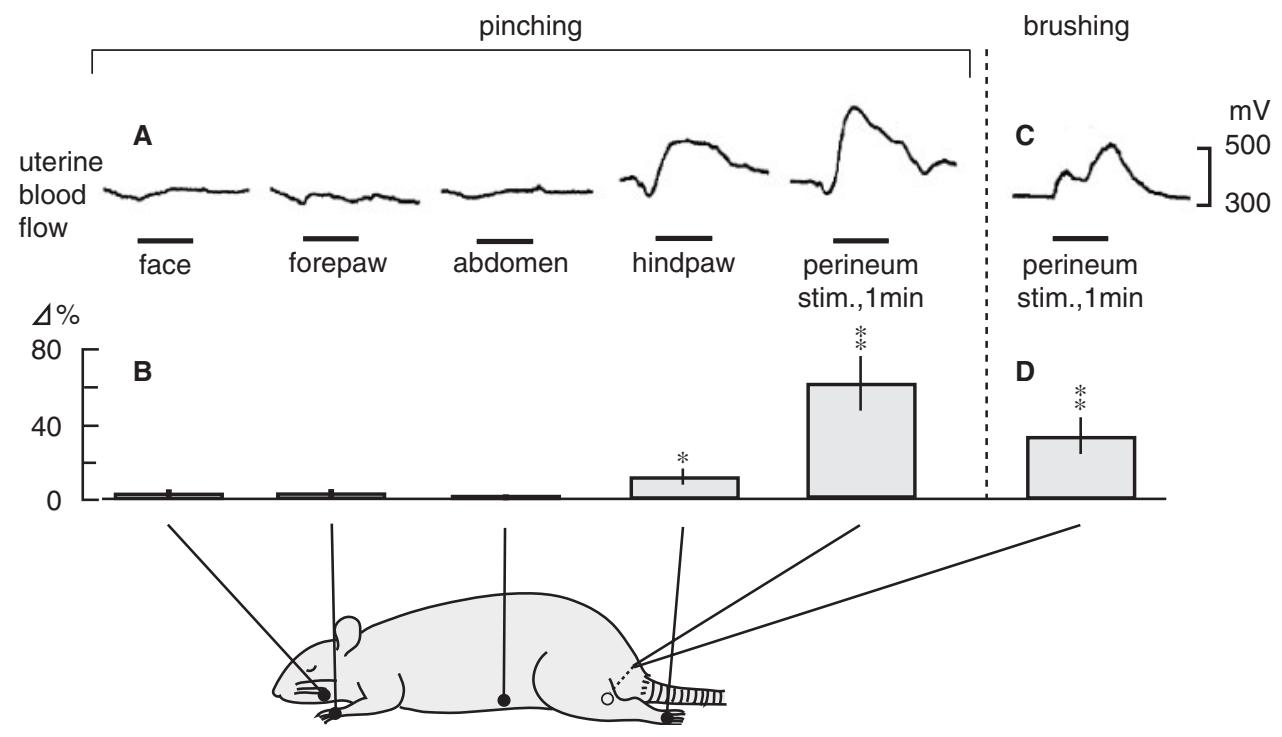

Figure 3. The effect of cutaneous stimulation of various areas on uterine blood flow in central nervous system-intact rats. (A and C) Sample recordings of uterine blood flow responses following pinching stimulation of various segmental areas of the body (A) or perineal brushing (C) for 1 min. (B) Summary of the responses of uterine blood flow to pinching of various skin areas and (D) brushing of the perineum. Changes in maximum responses of uterine blood flow during stimulation were calculated. Each column and vertical bar represent the mean $\pm \operatorname{SEM} .{ }^{*} P<0.05$; $* * P<0.01$; significantly different from pre-stimulus control values, using $t$-test. Modified from Hotta et al. (23).

increases in UBF was not always accompanied by arterial pressure responses.

\section{Parasympathetic Cholinergic Vasodilators are the Efferent Pathway of the UBF Response}

Blood flow of the uterus is known to be regulated reciprocally by parasympathetic (pelvic) cholinergic vasodilators and sympathetic (hypogastric) adrenergic vasoconstrictors in rats (24). Severance of bilateral pelvic nerves completely abolished the increase in UBF elicited by cutaneous stimulation, while the severance of bilateral hypogastric nerves alone did not significantly influence the responses of UBF. These results indicate that pelvic nerves are efferent paths for the increase in UBF. In fact, uterine pelvic parasympathetic efferent activity was increased by perineal pinching (Fig. 4); therefore, the UBF response was a reflex response via the activation of pelvic efferent nerves.

\section{Reflex Center for the UBF Responses is in the Spinal Cord}

These cutaneous stimulation-induced responses of blood flow and pelvic efferent nerve activity still existed and were even augmented, after acute spinalization at the first cervical level; therefore, the reflex center for these responses is in the spinal cord. The somato-uterine blood flow reflex showed pronounced segmental organization, since only cutaneous stimulation of the hindpaw and perineal area, with perineal predominance, produced these reflexes significantly. These segments of cutaneous afferents are close to the sixth lumbar and first sacral segments, from which pelvic efferent preganglionic fibers emerge in the spinal cord in rats (25).

\section{Summary of the reflex pathway for the UBF response}

We conclude that the cutaneous mechanical sensory stimulation can regulate UBF by a segmental spinal reflex mechanism via pelvic parasympathetic cholinergic vasodilators (Fig. 5). Perineal stimulation inducedincrease in the UBF was elicited either by innocuous brushing or noxious pinching, suggesting that the response was elicited by excitation of somatic groups II, III and IV afferent nerves.

\section{CGRP-Related Vasodilative Regulation of Skeletal Muscle Blood Flow (MBF)}

\section{Electro-acupuncture Affects MBF}

Noguchi et al. (26) examined effects of electro-acupuncture stimulation of the hindpaw on blood flow of the biceps femoris muscle in anesthetized rats. Electroacupuncture stimulation produced biphasic responses in muscle blood flow (MBF): an initial increase and then a slight decrease, with a concomitant increase in arterial blood pressure. The increase in MBF is due to passive response to a systemic reflex pressor response and the decrease in MBF is due to reflex activation of muscle sympathetic activity. 


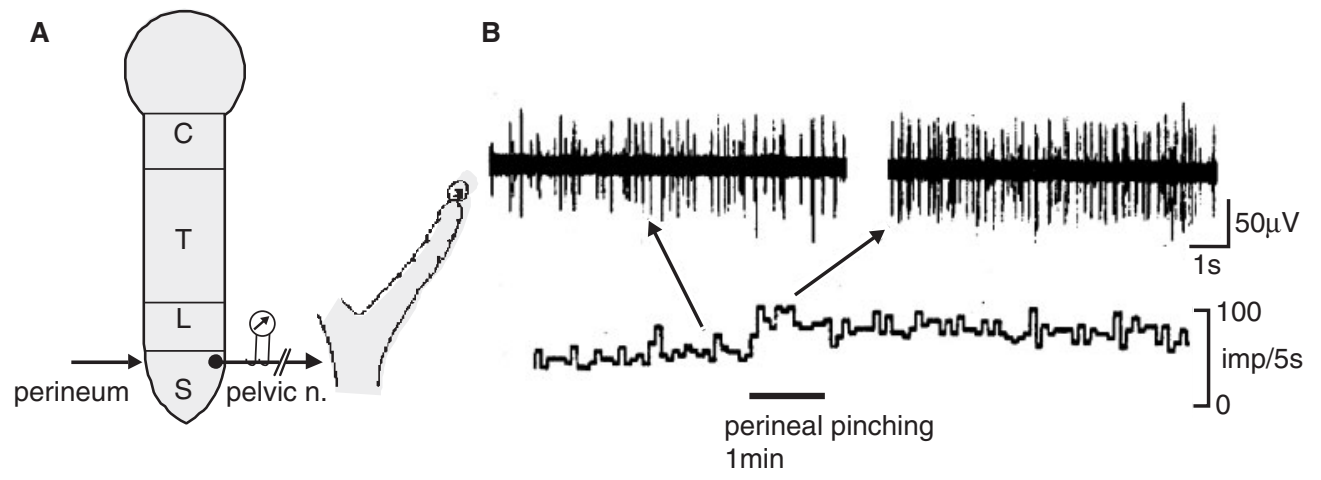

Figure 4. Effects of cutaneous stimulation on uterine pelvic efferent nerve activity in central nervous system-intact rats. (A) Schematic diagram of experimental procedures for perineal stimulation and recording of uterine pelvic efferent nerve activity. (B) Sample recordings of uterine pelvic efferent nerve responses following perineal pinching. Modified from Hotta et al. (23).

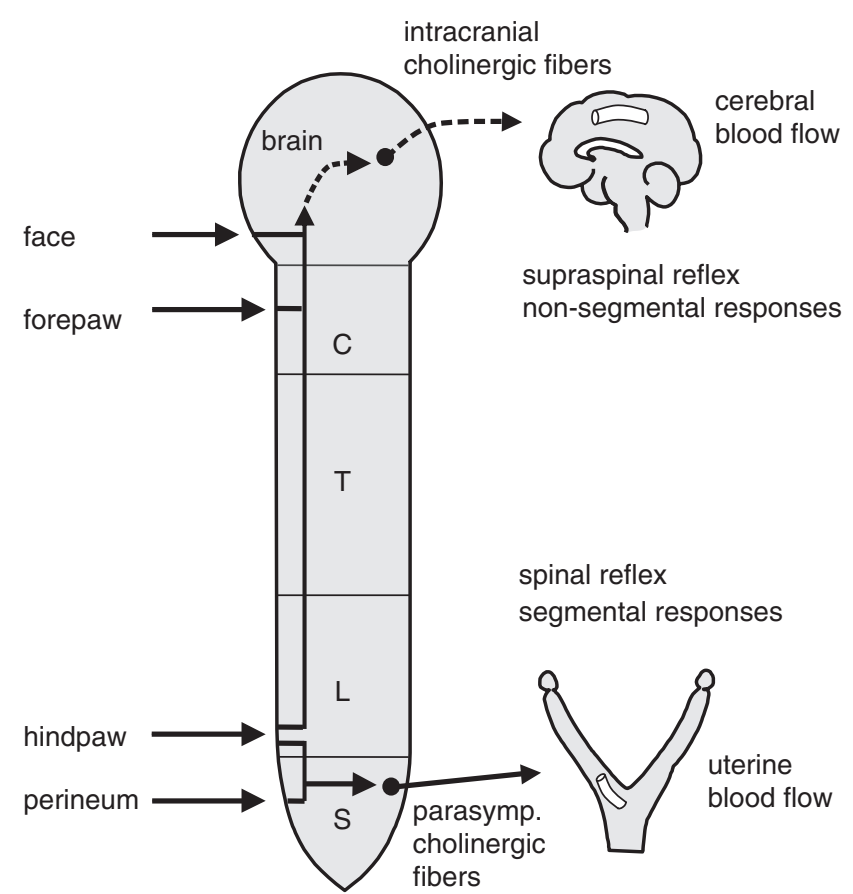

Figure 5. Schematic diagram of the reflex pathways for cerebral cortical and uterine blood vessels.

\section{Antidromic Activation of Somatic Afferent Nerves Increases MBF}

In acupuncture treatment for localized muscle ache, it is very likely that acupuncture stimulates muscle afferent fibers, either antidromically or by an axon reflex manner and releases CGRP. This vasodilative substance may then produce vasodilation and subsequent increases in the blood flow of local muscles. This possibility has long been proposed, but there has been no experimental proof that CGRP from the nociceptive afferent nerve terminals in muscles contribute to vasodilation of skeletal muscles. Recently, CGRP-related antidromic vasodilative action in muscles was demonstrated in rats by Sato et al. (27). When stimulus parameters were adequate to excite unmyelinated C (or group IV) afferent fibers, stimulation of the third to fifth lumbar dorsal roots produced an increase in blood flow in the biceps femoris muscle (Fig. 6). MBF increase was observed in $\sim 50 \%$ of the trials in which the L3 dorsal root was stimulated and in all trials in which the L4 or L5 dorsal roots were stimulated. This response was only obtained by stimulation of the dorsal root ipsilateral but not contralateral, to the biceps femoris muscle. Mean arterial pressure was not influenced by stimulation of these dorsal root segments.

The dorsal root stimulation-induced increase in $\mathrm{MBF}$ was totally abolished after topical application of $10^{-4} \mathrm{Mh}$ CGRP (8-37), a CGRP receptor antagonist (Fig. 6C). Thin afferent fibers containing CGRP exist in skeletal muscles (28) and CGRP is released in the hindlimb soleus muscles by excitation of the afferents in L4-L5 dorsal roots (29). This evidence indicates that antidromic vasodilation in skeletal muscles by stimulation of unmyelinated $\mathrm{C}$ afferents in dorsal roots is essentially mediated by the release of CGRP from afferent nerve terminals.

\section{Conclusion and Remarks}

As introduced in this article, the reflex neural mechanisms of the acupuncture effects on regional blood flow in various organs, such as the cerebral cortex, uterus and skeletal muscles, using anesthetized animals from which emotional factors have been eliminated, have been clarified (Fig. 7). Manual acupuncture needle stimulation of the skin and muscles has been reported to excite somatic afferent nerve fibers belonging to all groups I-IV fibers in rats (30). The afferent pathway of the increase in cerebral blood flow elicited by acupuncture-like stimulation is composed of somatic groups III and IV fibers and that in uterine blood flow is composed of somatic groups II-IV fibers. Efferent pathways of the increase in 
A

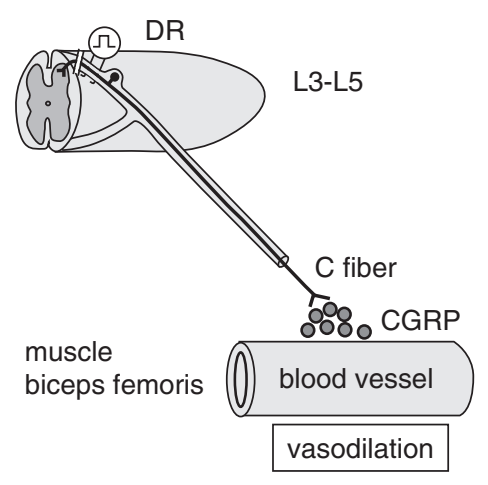

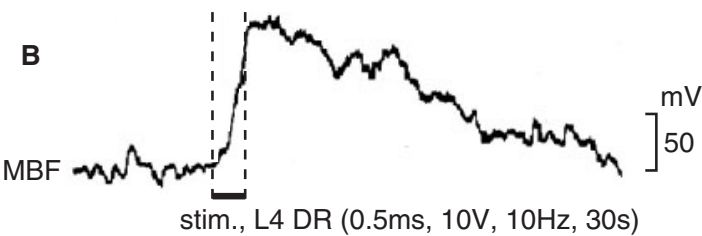

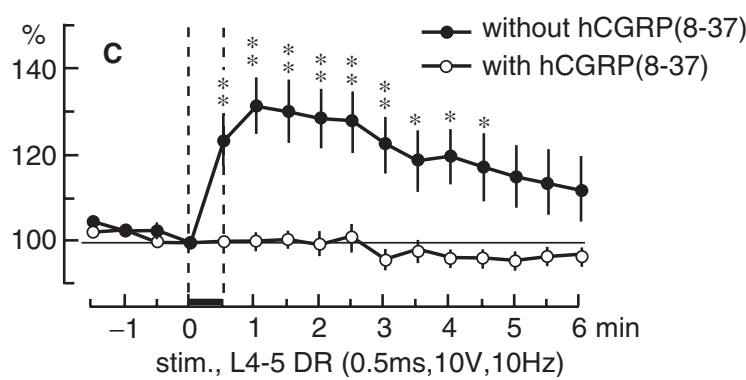

Figure 6. The effect of antidromic stimulation of the unilateral dorsal roots (DR) of lumbar segments on ipsilateral MBF. (A) Schematic diagram of CGRP-related antidromic vasodilative action in muscles. (B) Sample recordings of blood flow of the biceps femoris muscle response to antidromic electrical stimulation of the L4 DR. Parameters of electrical stimulation are indicated. (C) The means and SEM of MBF responses to stimulation of L4-L5 DR before (closed circles) and after (open circles) topical application of hCGRP (8-37) $\left(10^{-4} \mathrm{M}\right)$. Changes in MBF were calculated every $30 \mathrm{~s}$ and are expressed as percentages of the pre-stimulus values just before stimulation (ordinate). The horizontal bar between the dashed vertical lines indicates the time during DR stimulation. Onset of electrical stimulation of DR is expressed as zero (abscissa). ${ }^{*} P<0.05 ;{ }^{* *} P<0.01$; significantly different from pre-stimulus control values. Modified from Sato et al. (27).

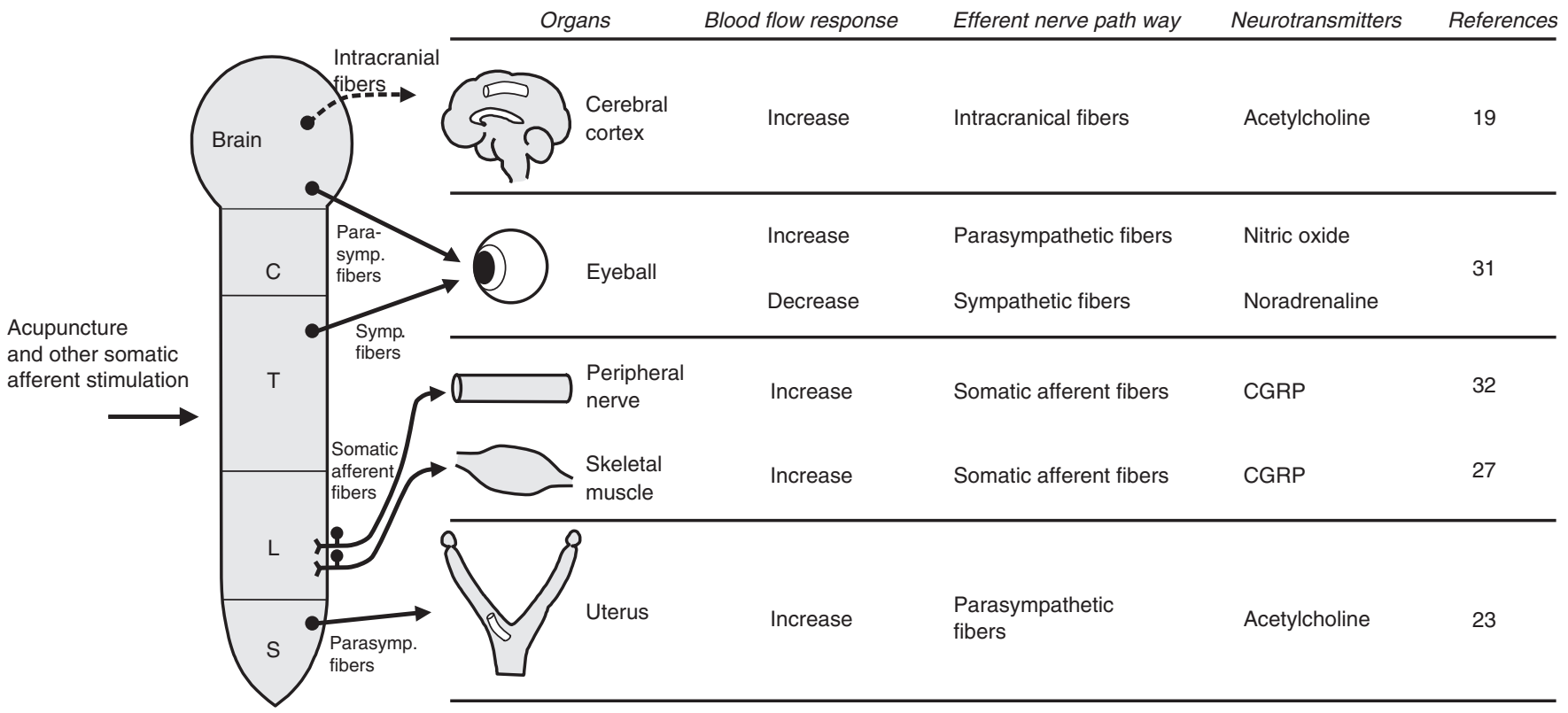

Figure 7. Schematic diagram showing neural mechanisms of blood flow responses in various organs induced by acupuncture and other somatic afferent stimulation.

CBF by acupuncture-like stimulation include intrinsic cholinergic vasodilators originating in the basal forebrain and those in uterine blood flow include pelvic parasympathetic cholinergic vasodilators. The reflex increases in cerebral blood flow are supraspinal reflexes characterized by non-segmental organization. On the other hand, the reflex response in uterine blood flow is a spinal reflex characterized by segmental organization. Regulation of regional blood flow by somatic afferent stimulation, which is based on somato-autonomic reflex mechanisms, have also been found in choroidal blood flow of the eyeball (31) (Fig. 7).

Skeletal muscle blood flow is also regulated by electroacupuncture stimulation, by reflex mechanism via central nervous system. Furthermore, it was found that the antidromic activation of group IV somatic afferent nerves produces vasodilative action in skeletal muscles via CGRP; this phenomenon does not require the presence 
of the central nervous system. Similar antidromic vasodilation in peripheral nerves has also been demonstrated (32) (Fig. 7).

The principles developed, based on these studies of anesthetized animals may be applicable to understanding the neural mechanisms of blood flow responses of various organs following clinical acupuncture.

\section{References}

1. Sato A, Sato Y, Schmidt RF. The impact of somatosensory input on autonomic functions. Rev Physiol Biochem Pharmacol 1997; 130:1-328.

2. Mann F. Reinventing Acupuncture, 2nd edn. London: ButterworthHeinemann, 2001.

3. Li P, Yao T. Mechanism of the Modulatory Effect of Acupuncture on Abnormal Cardiovascular Functions. Shanghai: Shanghai Medical University Press, 1992.

4. Stux G, Pomeranz B. Basics of Acupuncture, 5th edn. Berlin: Springer-Verlag, 2003.

5. Sato A, Sato Y, Suzuki A, Uchida S. Reflex modulation of gastric and vesical function by acupuncture-like stimulation in anesthetized rats. Biomed Res 1994;15:59-65.

6. Sato A, Sato Y, Uchida S. Reflex modulation of visceral functions by acupuncture-like stimulation in anesthetized rats. International Congress Series 2002;1238:111-23.

7. Ma S-X. Neurobiology of Acupuncture: Toward CAM. eCAM 2004;1:41-7.

8. Sato A, Sato Y, Suzuki A, Uchida S. Neural mechanisms of the reflex inhibition and excitation of gastric motility elicited by acupuncture-like stimulation in anesthetized rats. Neurosci Res 1993;18:53-62.

9. Sato A, Sato Y, Suzuki A. Mechanism of the reflex inhibition of micturition contractions of the urinary bladder elicited by acupuncture-like stimulation in anesthetized rats. Neurosci Res 1992;15:189-98.

10. Ohsawa H, Okada K, Nishijo K, Sato Y. Neural mechanism of depressor responses of arterial pressure elicited by acupuncture-like stimulation to a hindlimb in anesthetized rats. J Auton Nerv Syst 1995:51:27-35.

11. Sato A, Sato Y, Suzuki A, Uchida S. Reflex modulation of catecholamine secretion and adrenal sympathetic nerve activity by acupuncture-like stimulation in anesthetized rat. Jpn J Physiol 1996:46:411-21.

12. Mori H, Uchida S, Ohsawa H, Noguchi E, Kimura T, Nishijo K. Electro-acupuncture stimulation to a hindpaw and a hind leg produces different reflex responses in sympathoadrenal medullary function in anesthetized rats. $J$ Auton Nerv Syst 2000;79:93-8.

13. Wong AMK, Su T-Y, Tang F-T, Cheng P-T, Liaw M-Y. Clinical trial of electrical acupuncture on hemiplegic stroke patients. Am J Phys Med Rehabil 1999;78:117-22.

14. Tian J. Research into the treatment of vascular dementia in China using traditional therapies. Age and Ageing 1998;27:247-50.

15. Yu J, Zhang X, Liu C, Meng Y, Han J. Effect of acupuncture treatment on vascular dementia. Neurol Res 2006;28:97-103.
16. Stener-Victorin E, Waldenström U, Andersson SA, Wikland M. Reduction of blood flow impedance in the uterine arteries of infertile women with electro-acupuncture. Hum Reprod 1996;11: 1314-17.

17. Chang R, Chung PH, Rosenwaks Z. Role of acupuncture in the treatment of female infertility. Fertil Steril 2002;78:1149-53.

18. Lundeberg $\mathrm{T}$, Hurtig $\mathrm{T}$, Lundeberg $\mathrm{S}$, Thomas M. Long-term results of acupuncture in chronic head and neck pain. The Pain Clinic 1988;2:15-31.

19. Uchida S, Kagitani F, Suzuki A, Aikawa Y. Effect of acupuncturelike stimulation on cortical cerebral blood flow in anesthetized rats. Jpn J Physiol 2000;50:495-507.

20. Adachi T, Meguro K, Sato A, Sato Y. Cutaneous stimulation regulates blood flow in cerebral cortex in anesthetized rats. Neuro Report 1990;1:41-4.

21. Sato A, Sato Y. Regulation of regional cerebral blood flow by cholinergic fibers originating in the basal forebrain. Neurosci Res 1992;14:242-74.

22. Akaishi T, Kimura A, Sato A, Suzuki A. Responses of neurons in the nucleus basalis of Meynert to various afferent stimuli in rats. Neuro Report 1990;1:37-9.

23. Hotta H, Uchida S, Shimura M, Suzuki H. Uterine contractility and blood flow are reflexively regulated by cutaneous afferent stimulation in anesthetized rats. J Auton Nerv Syst 1999;75:23-31.

24. Sato Y, Hotta H, Nakayama H, Suzuki H. Sympathetic and parasympathetic regulation of the uterine blood flow and contraction in the rat. J Auton Nerv Syst 1996;59:151-8.

25. Gabella G. Autonomic nervous system. In: Paxinos G (ed). The Rat Nervous System, Vol. 2 Hindbrain and Spinal Cord. Sydney: Academic Press, 1985, 325-53.

26. Noguchi E, Ohsawa H, Kobayashi S, Shimura M, Uchida S, Sato Y. The effect of electro-acupuncture stimulation on the muscle blood flow of the hindlimb in anesthetized rats. J Auton Nerv Syst 1999; 75:78-86.

27. Sato A, Sato Y, Shimura M, Uchida S. Calcitonin gene-related peptide produces skeletal muscle vasodilation following antidromic stimulation of unmyelinated afferents in the dorsal root in rats. Neurosci Lett 2000;283:137-40.

28. Edvinsson L, Gulbenkian S, Wharton J, Jansen I, Polak JM. Peptide-containing nerves in the rat femoral artery and vein: An immunocytochemical and vasomotor study. Blood Vessels 1989;26:254-71.

29. Sakaguchi M, Inaishi Y, Kashihara Y, Kuno M. Release of calcitonin gene-related peptide from nerve terminals in rat skeletal muscle. J Physiol 1991;434:257-70.

30. Kagitani F, Uchida S, Hotta H, Aikawa Y. Manual acupuncture needle stimulation of the rat hindlimb activates groups I, II, III and IV single afferent nerve fibers in the dorsal roots. Jpn $J$ Physiol 2005;55:149-55.

31. Shimura M, Uchida S, Suzuki A, Nakajima K, Aikawa Y. Reflex choroidal blood flow responses of the eyeball following somatic sensory stimulation in rats. Autonomic Neurosci 2002;97:35-41.

32. Sato A, Sato Y, Uchida S. Blood flow in the sciatic nerve is regulated by vasoconstrictive and vasodilative nerve fibers originating form the ventral and dorsal roots of the spinal nerves. Neurosci Res 1994;21:125-33.

Received January 11, 2007; accepted April 11, 2007 


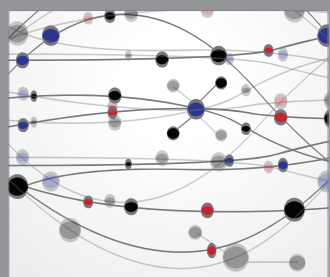

The Scientific World Journal
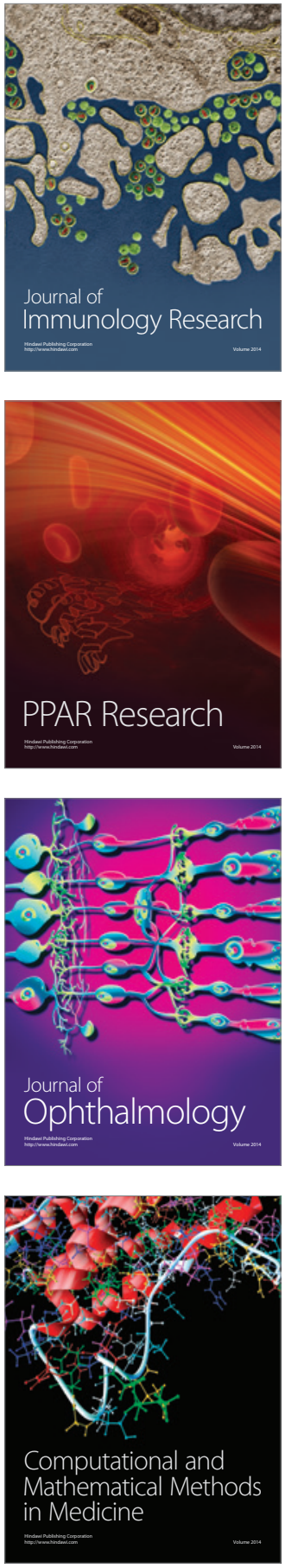

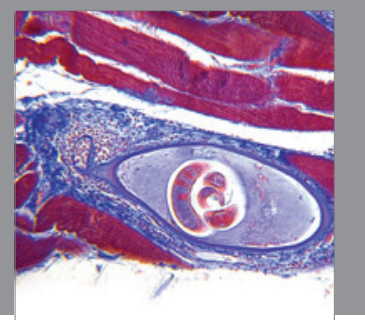

Gastroenterology

Research and Practice
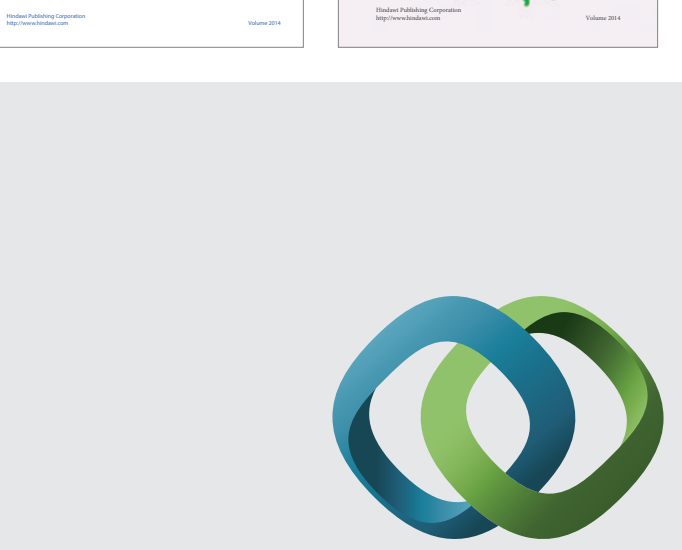

\section{Hindawi}

Submit your manuscripts at

http://www.hindawi.com
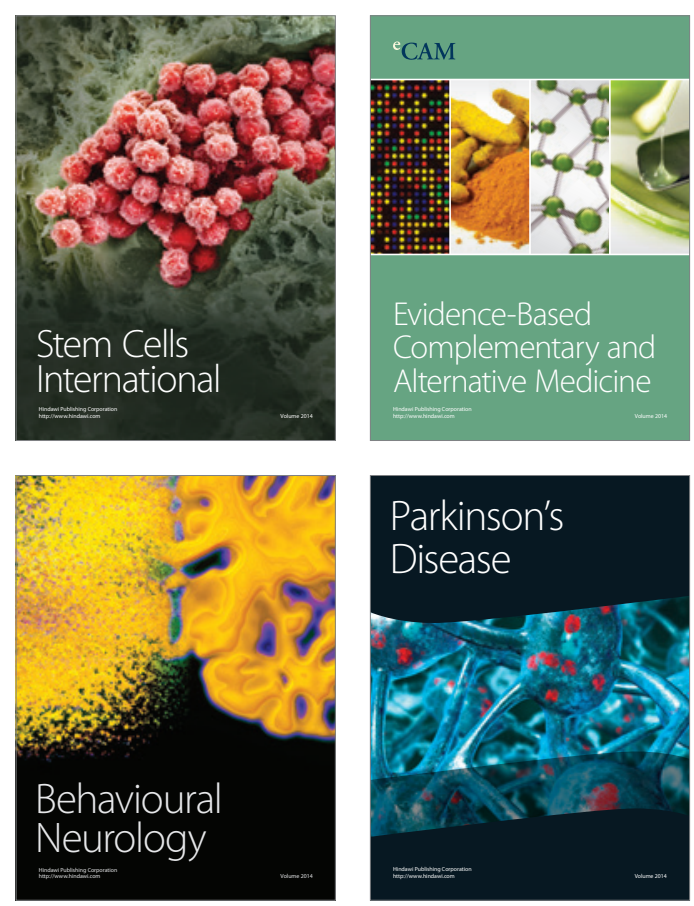

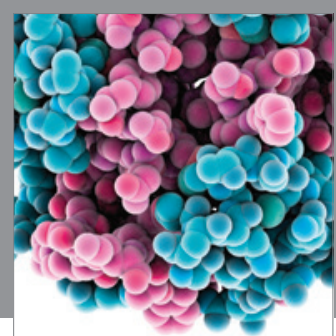

Journal of
Diabetes Research

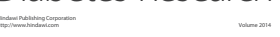

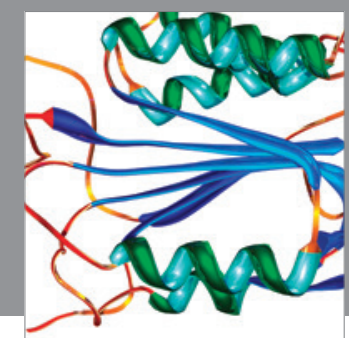

Disease Markers
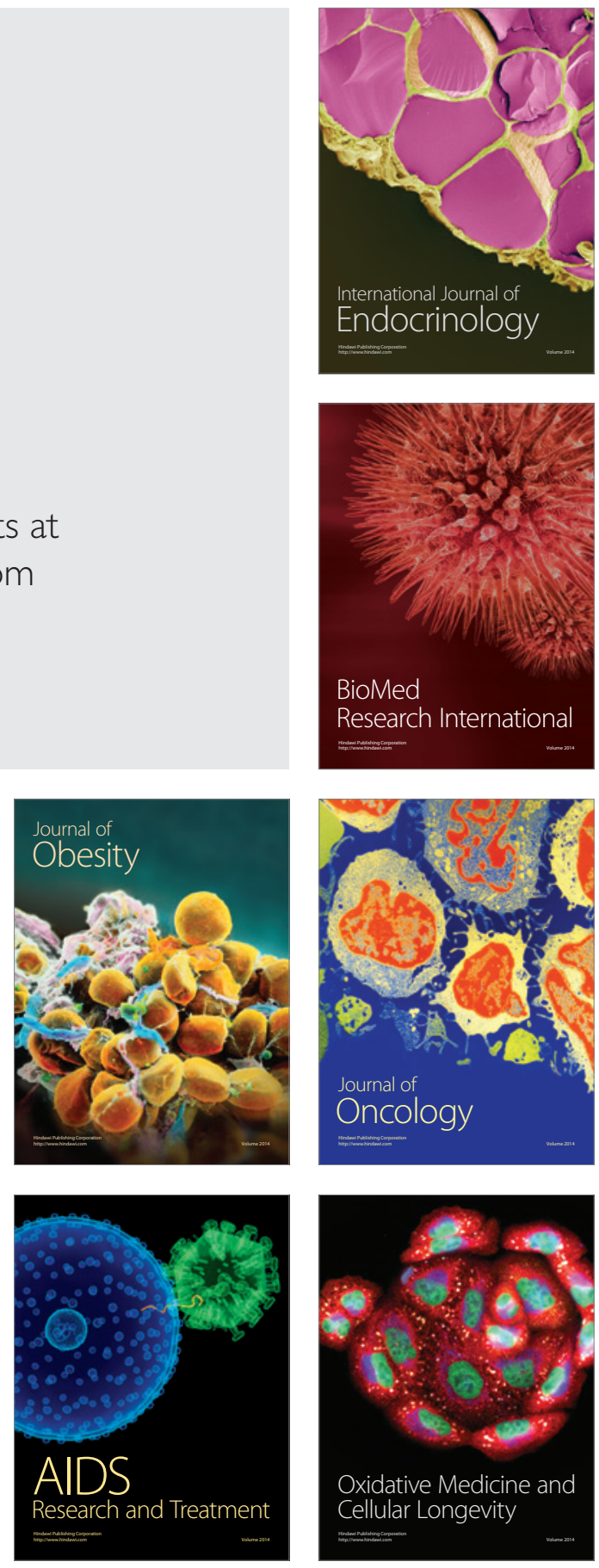